\title{
Gelotophobia in practice and the implications of ignoring it
}

\author{
Tracey Platt \\ University of Wolverhampton \\ T.Platt@wlv.ac.uk
}

\section{René T. Proyer}

Zurich University

r.proyer@psychologie.uzh.ch

\author{
Jennifer Hofmann \\ Zurich University \\ j.hofmann@psychologie.uzh.ch
}

\author{
W. Larry Ventis \\ College of William and Mary \\ wlvent@wm.edu
}

\begin{abstract}
Over 85 empirical articles investigating the fear of being laughed at have been published. Still, the question "why bother with another inter-individual differences variable?" arises. This quantitative paper based on 240 people, aims to show why gelotophobia has been widely neglected in therapeutic settings and why therapists may not have come across gelotophobes in their practice. Second, examples of extreme case studies involving gelotophobe's perspective on treatment, exploring the practice and challenges arising in treatments will be given. Finally, an argument why there is a need for the inclusion of gelotophobia awareness for schools and for bullying interventions is proposed. Conclusions are drawn suggesting the importance of inclusion of this phenomenon.
\end{abstract}

Keywords: fear of being laughed at, psychoanalysis, counselling, bullying, laughter, humour.

Smiling and laughter are integral to everyday social interactions and communication. They are the non-verbal way we express that we are experiencing enjoyable emotional states (Ekman, Davidson, \& Friesen, 1990). When we smile at someone, or laugh with somebody, we naturally assume people are attributing positive intent. However, laughter can also be used to 
mock, ridicule and demean. So not all smiles or laughter are friendly (see Ruch, Hofmann, \& Platt 2013). This dual signalling function creates ambiguity for those interpreting the context and, for some, differentiating the meanings is problematic. Such people often have a negative attribution bias (Hofmann, Platt, \& Ruch, 2015; Platt, 2008; Ruch, Altfreder, \& Proyer, 2009). They assume that all laughter is "bad" laughter, which elicits in them feelings of fear and shame. This inability has been found to go along with a fear of being laughed at, commonly known as 'gelotophobia' (Platt \& Ruch, 2009; Ruch, Hofmann, Platt, \& Proyer, 2014; Titze, 2009).

Although everyone has been laughed at and it is unpleasant, usually there are no long lasting negative effects. Post-event, people typically do not change to believing that all smiling and laughter is ridiculing them, but gelotophobes do. Therefore, this construct is important in the explanation of the misperception of the benevolent nature of humour and laughter.

From clinical observations of gelotophobia (e.g., Titze, 1996), the investigation of the disorder moved to samples of the normal population. Ruch and Proyer (2008a, 2008b) derived cut-off points for levels ranging from 'no...' to 'extreme gelotophobia'. They found that the fear of being laughed at was still prevalent among the normal population and concluded that it should be seen as an individual difference variable at a sub-clinical level.

Gelotophobia, namely, the fear of being laughed at (see Ruch et al. 2014) has been found in over 90 samples tested on every continent (Proyer, Ruch, Ali, Al-Olimat et al. 2009). Those affected misperceive smiling, laughter and laughter-related cues as being aversive and negative. They believe that laughter is a weapon that puts them down, and, overall, they experience laughing with as being laughing at and they believe that any laughter is negatively directed at them. In this regard, they believe they are not able to keep up with other people's humour, despite regarding themselves as producing it (see Ruch et al. 2014 for a review).

Gelotophobes' personality traits are mainly assessed as being introvert-neurotic, with the additional tendency to be lower in openness to experience or, higher in psychoticism (e.g., Radomska \& Tomczak 2010; Rawlings et al. 2010; Ruch et al. 2008; Ruch \& Proyer 2009). Such personality trait configuration commonly goes along with social anxiety, timidity, shame-proneness and other variables relating to social withdrawal and anxiety (e.g., Corrs, 2004; Crazier, 1979; Sternberger, Turner, Beidel, \& Calhoun, 1995).

Hartenberg (1901) first made a link between timidity and the fear of ridicule, which subsequently was dropped from further specific investigations. Yet, all research as to whether gelotophobia was just "social phobia re-packaged" showed that social phobia did not explain all the variance and that the fear of being laughed at could not be fully accounted for by measures of social phobia (Carretero-Dios et al., 2010; Edwards et al., 2010, Weiss et al., 2012). This became clarified when investigating those at the extreme end of the continuum, where it was found that components or facets of coping with derision (by control, withdrawal, internalising), disproportionate negative responses to being laughed at, and paranoid sensitivity to anticipated ridicule could be found (Platt, Ruch, Hofmann \& Proyer, 2012). These factors clearly showed where the overlap with social phobia lays and what remained were unique qualities of gelotophobia.

The aim of this paper is to argue that gelotophobia is an important personal/social issue and should be considered in its own right both in practice and research. Special consideration is seen as desirable for counsellors, who may never have experienced a gelotophobe or who have clients presenting with behaviours of social phobia but who are not responding to therapy. The topic will be explored from three perspectives. 


\section{Perspective 1: Therapy as the barrier to healing?}

Many clinical psychologists, psychotherapists, psychiatrists dealing with patients, when questioned, state that they doubt the existence of gelotophobia and that the dynamics associated with the concept do not manifest into being a pathological problem; largely because, if it had, they would have come across it (see Platt 2013). This rather subjective perspective is basically true. Firstly, as the condition is not represented in the Diagnostic and Statistical Manual of Mental Disorders, it means anyone presenting with a diagnosis will have the wrong diagnosis, perhaps depression or social phobia for example, or secondly the practitioner may not have actually had anyone directly presenting with issues relating specifically to being laughed at. However, by exploring the role of the therapy and therapist, we will show how the therapeutic setting may in fact be the barrier.

Taking a psychoanalytic perspective (though we do not claim that gelotophobia can be exclusively explained in this context), we theorise how gelotophobia has been noticeable by its absence in clinical practice. Transference in psychoanalysis is the phenomenon of transferring feelings and thoughts one has toward significant others in one's life to the person of the analyst (Freud 1912). Freud argued that the heart of psychoanalysis depended on interpreting the transference. However, transference is often thought to be Freud's emphasis, and the advocates of other approaches simply chose to focus on other things (Ehrenreich 1989). It is not the case that other approaches do not recognise transference. It is just that it is not as critical to their therapies.

Once in therapy the analysand has to free associate, meaning they have to suspend their "editing functions" as best they can. This is not something easily accomplished and is highly variable between clients (Temerlin 1956). However, over a period of weeks, months, or even years, they approximate this goal, revealing very extensive and personal fantasies, wishes, and fears.

To create an environment for the client to free associate, the therapist considers the physical arrangement of the clinical setting. The patient lies on a couch, with the psychoanalyst situated behind. It is in this position that the client is asked to say aloud whatever comes to mind, presumably a great deal of which is highly personal information that one might otherwise edit out. It will come as no surprise that clients commonly wonder what the therapist thinks about them. This form of inhibition may even threaten the therapy (Lawrence 2003).

Assuming that the analyst does not respond to direct questions, which they do not, the client does what virtually anyone would do. They project their expectations onto the analyst. If they have been treated in a nurturing, caring manner in their past, this will likely be what they expect from, or transfer onto, the analyst. However, if they were neglected or abused, then it is that expectation which is likely to be transferred onto the analyst. So, the task and the physical layout, induce this transference of feelings about important others onto the analyst, and, hopefully, this reveals significant unconscious feelings and expectations.

Putting this therapeutic relationship into the context of someone with the fear of being laughed at, it becomes clear why many therapists report, even after many years in practice, to having not treated gelotophobes, even doubting its existence (Ventis \& Platt, 2014). There may however be a plausible reason. To convey their good intentions to their client, a therapist would smile - this may trigger gelotophobic responses in those who come seeking help. Naturally, a reassuring smile would be usual for a clinician, meeting a new client in an initial psychotherapy session. In addition to gaining some clinically usable information, virtually anyone in this position is hoping to establish positive rapport. One prominently relied upon behavioural signal of benign intent is at least an occasional pleasant smile. However, this is exactly the thing the client is extremely sensitised to, as well as a potential harbinger of 
rejection or contempt (Hofmann, Platt, Ruch \& Proyer 2014). So, for a gelotophobe, a wellmeaning, smiling therapist, in the initial session, is highly likely to be alienating, and possibly frightening. This may explain why therapists often state that they have never treated gelotophobes in their practice. Independent of the type of therapy-reassuring smiles and humorous remarks may lead to a breakdown of the therapeutic relationship with a gelotophobe in the early stage of a treatment (leading them to drop out).

\section{Perspective 2: Evidence from case studies}

The account of psychoanalysis was not a digression because gelotophobes are highly likely to transfer their intense fear of being laughed at into the psychotherapy session. The therapist's well-intentioned smile, at the first session could well result in the client being frightened away. In a study of 2,551 cases drawn from 17 community mental health facilities, the authors found that $40.8 \%$ of the cases failed to return after the first interview (Sue, McKinney, \& Allen 1976). How many of those dropouts were gelotophobes? This we cannot know for sure, but it is argued that a certain portion might be due to gelotophobes having felt laughed at in the therapeutic setting.

An online website dedicated to the assessment of gelotophobia gathered information on gelotophobes and allowed the investigation whether gelotophobes actually considered going to a therapist and, if they had, whether the treatment had been successful. Some excerpts are given that help elucidate these and are detailed below.

\section{Method}

\section{Participants}

High scoring gelotophobes were invited to complete an online case-study $n=240$ participants. The sample consisted of $56.2 \%$ males, $37.3 \%$ females and $6.5 \%$ non-respondents, with ages ranging from 18 to 63 years $(M=25.1, S D=11.4)$.

\section{Instruments}

The GELOPH $<15>$ (Ruch \& Proyer 2008b) is a questionnaire assessing the level of the fear of being laughed at (i.e., gelotophobia) consisting of fifteen items in a four-point answer format $(1=$ strongly disagree; $2=$ moderately disagree; $3=$ moderately agree; $4=$ strongly agree). A large variety of studies have supported the high internal consistency, stability (see Ruch et al. 2014), and validity of the GELOPH $<15>$. The English translation sample (Platt $e t$ al. 2009) yielded a good reliability $(\alpha=.90)$ and this sample is highly consistent $(\alpha=.91)$.

The Structured Gelotophobia Interview-Written Experimental Form (Platt \& Ruch 2007) is an in depth assessment of the fear of being laughed at aimed at covering the relevant components of the causes and consequences of gelotophobia model presented by Ruch and Proyer (2008a). The SGI contains a list of 20 questions (e.g., regarding the onset of the fear of being laughed at, typical ways of dealing with it, thoughts, emotions and actions while being laughed at) that are presented in a written format. The answers of the participants to some of the questions are coded utilising a coding scheme developed on the basis of a content analysis on the responses to the open questions suggested by two independent raters. 


\section{Procedure}

The percentage of gelotophobia, worldwide is very limited (see Platt \& Forabosco, 2012). Making the problem of recruitment more difficult is their tendency to withdraw from society and face-to-face social interactions. Therefore, to test a sample that had a large enough distribution within each group, a novel way of engaging enough gelotophobes was needed. With this in mind, a website dedicated to gelotophobia was set up for data collection. The research was conducted in accordance with the Ethics Committee of the University where the researchers were based and in accordance with the APA ethical considerations. In the introduction page of the website, participants were told that clicking on the "next" button, there were also consenting to continue with the study and this could be terminated at any time. Feedback was offered to those completing the study and leaving contact details.

\section{Case study results}

Participant's gelotophobia scores ranged from 1.20 to 4.00 and the distribution statistics indicate that due to the selection process, there were more high scorers in the sample than usual, leading to a higher mean and higher standard deviation $(M=3.28, S D=0.48)$.

The pre-defined cut off points for level of gelotophobia was applied and showed that $2.1 \%$ $(n=5)$ had scores $<1.99$ in the GELOPH $<15>$ (Ruch \& Proyer 2008b) and thus classified as having no fear. Those participants classified as having borderline gelotophobia (scores between 2 and 2.49), 5.8\% $(n=14)$ and 8.3\% $(n=20)$ had slight gelotophobia (scores between 2.5 and 2.99). The majority of the sample consisted of $48.3 \%(n=116)$ with marked level of gelotophobia (scores between 3 and 3.49). Finally, 35.0\% $(n=85)$ of the participants had extreme fear of being laughed (scores $>3.5$ ).

In response to the question "have you ever sought help, successful or otherwise for gelotophobia", of the 240 participants, 173 replied "no" to the question. In Table 1 examples of the types of replies, the level of gelotophobia, and some demographic information on the case study participants are given. 


\begin{tabular}{|c|c|c|}
\hline $\begin{array}{c}\text { G. } \\
\text { Score }\end{array}$ & Demographics & "No" Reasons \\
\hline 3.47 & $\begin{array}{l}\text { Youth (18-20) } \\
\text { American male }\end{array}$ & $\begin{array}{l}\text { I have not. I refuse to try therapy and I refuse to tell my parents } \\
\text { anything about my life. Why would I? To give them a good laugh at my } \\
\text { expense? I don't even think my friends really care or have noticed }\end{array}$ \\
\hline 3.53 & $\begin{array}{l}\text { Young adult (25-30) } \\
\text { British male }\end{array}$ & No. I don't want anyone to think I am less than what I already am \\
\hline 3.80 & $\begin{array}{l}\text { Young adult }(25-30) \\
\text { American female }\end{array}$ & $\begin{array}{l}\text { I personally feel that this disorder is silly, and I am too ashamed to seek } \\
\text { help for it }\end{array}$ \\
\hline 3.27 & Female (30-35) Irish & $\begin{array}{l}\text { I haven't sought any kind of help yet, because I don't even think how } \\
\text { they could help }\end{array}$ \\
\hline $\begin{array}{c}\text { G. } \\
\text { Score }\end{array}$ & Demographics & "Yes but fai \\
\hline 3.27 & $\begin{array}{l}\text { Youth (18-20) } \\
\text { American male }\end{array}$ & Yes but I got rejected from therapy. \\
\hline 3.73 & $\begin{array}{l}\text { Youth (18-20) } \\
\text { American male }\end{array}$ & $\begin{array}{l}\text { Yes from a school counsellor (unsuccessful) though at the time she just } \\
\text { thought of it as a minor social problem }\end{array}$ \\
\hline 3.47 & $\begin{array}{l}\text { Young (20-25) } \\
\text { Iranian female }\end{array}$ & I sought help but it never worked for me \\
\hline 3.60 & $\begin{array}{l}\text { Young (20-25) } \\
\text { British female }\end{array}$ & $\begin{array}{l}\text { I'm on medication for depression and have received counselling but I've } \\
\text { always imagined there was more to my diagnosis, my fearing laughter } \\
\text { was always hidden. }\end{array}$ \\
\hline 3.33 & $\begin{array}{l}\text { Mid-aged (39-45) } \\
\text { British female }\end{array}$ & $\begin{array}{l}\text { Along with other issues, I have had counselling and extensive } \\
\text { psychotherapy. The therapist tried role-playing although I explained } \\
\text { that this was a situation I found humiliating and that actually made my } \\
\text { feelings worse for around } 3 \text { years. Psychotherapy helped to some extent } \\
\text { in that I can understand why I react this way, but it hasn't helped me } \\
\text { overcome the issues and was very expensive. }\end{array}$ \\
\hline
\end{tabular}

Table 1. Gelotophobia scores, demographic information and replies to case-study therapy question $^{1}$

Table 1 shows the different reasons given as to why people did not seek therapy or why they thought that the therapy had failed to help. Two distinct categories seem to develop, those who never tried and those who sought help but found it unsuccessful. The reasons for not seeking help clearly relates to the concern that if this condition was brought to others attention, it would induce shame or elicit laughter. These people often do not believe such therapy would help them. Those who sought therapy but failed relate situations where they feel that their fear of being laughed at has been trivialised or not taken seriously.

As we have argued, the very nature of a therapeutic setting may be a difficult environment and well-meaning smiling therapists reassuring their clients may be triggering gelotophobic beliefs that telling the therapist will belittle them and make them objects of derision. 


\section{Perspective 3: Gelotophobia in school therapy}

Several authors have argued that gelotophobia has a place in practice. One of the most recent examples is Bledsoe and Baskin's (2014) article on recognising student fear in the classroom. There, the authors also acknowledge the important role gelotophobia may have in such a setting by saying (about the student fearing being laughed at):

[...] Unable to view laughter's positive characteristics (e.g., community-building, tensionrelieving), such students perceive classroom laughter as threatening and shame-inducing and may demonstrate such nervous behaviours as fidgeting and looking down when humorous comments are made by students or the professor. They often appear overly quiet, and are unable to engage in even minimal discussions. A teacher who assumes that laughter is the best medicine may actually be contributing to the fear-based responses of some students suffering from gelotophobia. (p. 34)

This is important when thinking of how gelotophobia manifests itself in the school setting and how others perceive those students. Greater levels of gelotophobia are associated with feelings of being a victim of bullying; this has been shown for six to nine-year olds (also in teacher ratings; Proyer, Neukom, Platt, \& Ruch 2012), for adolescents (also in peer-ratings; Führ 2010; Proyer, Meier, Platt, \& Ruch 2013), and adults (Platt et al. 2009). Hence, considering the role of laughter (e.g., the difference between laughing at and laughing with), this may be important for school counsellors and teachers.

Programs directed at increasing the awareness of the beneficial, but also potentially harmful and/or threatening aspects of laughter may be helpful to prevent problems for students with gelotophobic inclinations. It should be noted that the question whether, in some cases, the belief of having been bullied may be a "false alarm" if the bullying event is laughter-related, especially if that student is a gelotophobe. We do not want to argue that the problem of bullying can be reduced to misperceptions. Contrary, we expect that the vast majority of these situations demand action in providing help to the victims, but there may be a number of cases in which "false alarm" was caused and the help would come in the way of therapy targeting gelotophobia. School counsellors and teachers need awareness of those who fear being laughed at and how to support them when interacting with others in the classroom as extreme cases show that those children undertaking school shootings often target those they felt laughed at them (Burgess, Garbarino, \& Carlson 2006).

There may be a need to extend existing bullying prevention programs to increase the awareness of the role laughter and the perception of laughter may play. For example, in the Olweus Bully Prevention Program (OBPP; Olweus, Limber, \& Mahalic 1999), there are clearly formulated rules that could be easily amended; e.g., "We will not laugh at others" or helping those that are being laughed at, or making others (e.g., teachers, counsellors) aware of students that are frequently being laughed at. Counsellors that are aware of these different ways of dealing with ridicule and being laughed at will also be able to provide better information to parents, other students, and teachers. Finally, counsellors should be aware that there might be certain comorbidities that come with greater levels of the fear of being laughed at. For example, Samson, Huber, and Ruch (2011) found that people with Asperger's syndrome display comparatively high levels of the fear of being laughed at and Weiss and colleagues (2012) found that certain personality disorders may be associated with greater levels of the fear of being laughed at. Overall, we would like to argue that knowledge about the way students and adults deal with laughter and ridicule may be of importance for counsellors when working with their clients. 


\section{Conclusion}

The fear of being laughed at pervades all sections of a population. Yet, the conflict between seeking help and avoiding the feeling of embarrassment and shame means those who need the help seldom receive it. The reassuring smiles of the therapist and the room designed to enable transference, can bar those seeking help. Constantly misreading signals, gelotophobes are often alienated and feel bullied. With the raised awareness bringing about a better understanding of the unique qualities of gelotophobia, which are different from those of social phobia, will allow for more specific and hopefully successful treatment of the gelotophobic patients. A further helpful solution would be the inclusion of diagnostic tools. This way those being treated would be easier to identify and to treat.

It will also help children deal with feelings of being bullied that can be addressed by school counsellors. If teachers and counsellors know about the problems some face with decoding smiles and laughter signals, they may be able to facilitate early treatment, which would prevent many of the life-long problems, such as isolation, paranoia, worry that gelotophobes face in their daily lives (Platt \& Ruch 2010).

\section{Compliance with ethical standards}

The authors declare that the research was conducted in the absence of any commercial or financial relationships that could be construed as a potential conflict of interest.

\section{Acknowledgements}

This publication benefited from the support of the Swiss National Centre of Competence in Research LIVES-Overcoming Vulnerability: Life Course Perspectives, which is financed by the Swiss National Science Foundation.

\section{Note}

${ }^{1}$ G-score $=$ Gelotophobia score measured by the GELOPH $<15>$. Typing and grammatical errors have been corrected; everything else is as written by the participant.

\section{References}

Bledsoe, T. S., \& Baskin, J. J. (2014). 'Recognizing student fear: The elephant in the classroom'. College Teaching 62 (1), pp. 32-41.

Burgess, A. W., Garbarino, C., \& Carlson, M. I. (2006). 'Pathological teasing and bullying turned deadly: Shooters and suicide'. Victims and Offenders 1 (1), pp.1-14.

Carretero-Dios, H., Ruch, W., Agudelo, D., Platt, T., \& Proyer, R. T. (2010). 'Fear of being laughed at and social anxiety: A preliminary psychometric study.' Psychology Science Quarterly 52 (1), p. 108.

Corrs, P. J. (2004). 'Reinforcement sensitivity theory and personality'. Neuroscience \& Biobehavioral Reviews 28 (3), pp. 317-332.

Crazier, W. R. (1979). 'Shyness as a dimension of personality'. British Journal of Social and Clinical Psychology 18 (1), pp. 121-128. 
Edwards, K. R., Martin, R. A., \& Dozois, D. J. (2010). 'The fear of being laughed at, social anxiety, and memories of being teased during childhood'. Psychology Science Quarterly 52 (1), pp. 94-107.

Ehrenreich, J. H. (1989). 'Transference: One concept or many?' Psychoanalytic Review 76 (1), pp. 37-65.

Ekman, P., Davidson, R. J., \& Friesen, W. V. (1990). 'The Duchenne smile: Emotional expression and brain physiology: II'. Journal of Personality and Social Psychology 58 (2), pp. 342- 353.

Freud, S. (1912). 'The dynamics of transference'. Essential Papers on Transference Analysis, pp. 5-17.

Führ, M. (2010). 'The applicability of the GELOPH $<15>$ in children and adolescents: First evaluation in a large sample of Danish pupils'. Psychology Science Quarterly 52 (1), pp. 60-76.

Hartenberg, P. (1901). Les Timides et la timidité (The socially anxious and social anxiety). Paris: Félix Alcan.

Hofmann, J., Platt, T., Ruch, W., \& Proyer, R. (2014). 'Individual differences in the decoding and responding to joy and contempt.' Sage Open.

Lawrence, W. G. (Ed.). (2003). Experiences in Social Dreaming. Karnac Books.

Olweus, D., Limber, S., \& Mahalic, S. F. (1999). Bullying prevention program. Center for the Study and Prevention of Violence, Institute of Behavioral Science, University of Colorado at Boulder.

Platt, T. (2008). 'Emotional responses to ridicule and teasing: Should gelotophobes react differently?' Humor: International Journal of Humor Research 21 (2), pp. 105-128.

Platt, T. (2013). Gelotophobia: Structure, Intensity Levels and Relations to Facially Expressed and Felt Emotions (published doctoral thesis). University of Zurich, Switzerland.

Platt, T. \& Ruch, W. (2007). The Structured Gelotophobia Interview-Written Experimental Form. Unpublished Questionnaire, University of Zurich, Switzerland.

Platt, T. \& Ruch, W. (2009). 'The emotions of gelotophobes: Shameful, fearful and joyless?' Humor: International Journal of Humor Research 22 (1-2), pp. 91-110.

Platt, T., Proyer, R. T., \& Ruch, W. (2009). 'Gelotophobia and bullying: The assessment of the fear of being laughed at and its application among bullying victims'. Psychological Science Quarterly 51 (2) pp. 135-147.

Platt, T. \& Ruch, W. (2010). 'A lifetime of the fear of being laughed at: An aged perspective'. Zeitschrift für Gerontologie und Geriatrie 43 (1), pp. 36-41.

Platt, T. \& Forabosco, G. (2012). 'Gelotophobia: The fear of being laughed at', in P. Gremigni (ed.), Humor and Health Promotion, Hauppauge, NY: Nova Science Publishers, pp. 229252.

Platt, T., Ruch, W., Hofmann, J., \& Proyer, R. T. (2012). 'Extreme fear of being laughed at: Components of gelotophobia'. The Israeli Journal of Humor Research: An International Journal 1, 86-106.

Proyer, R. T, Ruch, W., Ali, N. S., Al-Olimat, H. S., Amemiya T., Adal, T. A., Ansari, S. A., Arhar, Š., Asem, G., Baudin, N., Bawab S., Bergen, D., Brdar, I., Brites, R., BrunnerSciarra, M., Carrell A., Carretero Dios, H., Celik, M., Ceschi, G., Chang, K, Chao-chih, L., Cheryomukhin, A., Chik, M. P. Y., Chlopicki, W., Cranney, J., Dahourou, D., Doosje, S., Dore, M., El-Arousy, N., Fickova, E., Führ, M., Gallivan, J., Geling, H., Germikova, L., Giedraityte, M., Goh, A., González, R. D., Guohai, C., Ho, S. K., Hrebícková, M., Jaime, B., Jun, W., Hertzberg Kaare, B., Kamble, S., Kazarian, S., Kerkkanen, P., Klementová, M., Kobozeva, I. M., Kovjanic, S., Kumaraswamy, N., Lampert, M., Levesque, M., Loizou, E., Loving, R. D., Lyttle, J., Machline, V. C., McGoldrick, S., Min, L., McRorie, M., Mõttus, R, Munyae, M. M., Navia, C. E., Nkhalamba, M., Pedrini, 
P. P., Petkova, M., Platt, T., Popa, D.-E., Radomska, A., Rashid, T., Rawlings, D., Rubio, V. J., Samson, A. C., Sarid, O., Shams, S., Sisokohm, S., Jakob, S., Snikhovska, I., Stephanenko, E. A., Stokenberga, L., Stuer, H., Tanoto, Y. S. R., Tapia, L., Taylor, J., Thibault, P., Thompson, A., Thörn, H., Toyota, H., Ujlaky, J., Vanno, V., van der Westhuizen, B., Wijayathilake, D., Wong, P. S. O., Wycoff, E. B, \& Yeun, E. J. (2009). 'Breaking ground in cross-cultural research on the fear of being laughed at (gelotophobia): A multi-national study involving 73 countries'. Humor: International Journal of Humor Research 22 (1-2), 253-279.

Proyer, R. T., \& Ruch, W. (2009). 'Intelligence and gelotophobia: The relations of selfestimated and psychometrically measured intelligence to the fear of being laughed at'. Humor: International Journal of Humor Research 22 (1-2), pp. 165-181.

Proyer, R. T., Meier, L. E., Platt, T. \& Ruch, W. (2013). 'Dealing with laughter and ridicule in adolescence: Relations with bullying and emotional responses'. Social Psychology of Education 16 (3), pp. 399-420.

Proyer, R. T., Neukom, M., Platt, T., \& Ruch, W. (2012). 'Assessing gelotophobia, gelotophilia, and katagelasticism in children: An initial study on how six to nine-year-olds deal with laughter and ridicule and how this relates to bullying and victimization'. Child Indicators Research 5 (2), pp. 297-316.

Radomska, A., \& Tomczak, J. (2010). 'Gelotophobia, self-presentation styles, and psychological gender'. Psychological Test and Assessment Modeling 52 (2), pp. 191-201.

Rawlings, D., Tham, T.A., \& Milner Davis, J. (2010). 'Gelotophobia, personality and emotion ratings following emotion-inducing scenarios'. Psychological Test and Assessment Modeling 52 (2), pp. 161-170.

Ruch, W., \& Proyer, R. T. (2008a). 'The fear of being laughed at: Individual and group differences in gelotophobia'. Humor: International Journal of Humor Research 21 (1), pp. 47- 67.

Ruch, W., \& Proyer, R.T. (2008b). 'Who is gelotophobic? Assessment criteria for the fear of being laughed at'. Swiss Journal of Psychology 67 (1), pp. 19-27

Ruch, W., \& Proyer, R. T. (2009). 'Who fears being laughed at? The location of gelotophobia in the PEN-model of personality'. Personality and Individual Differences 46 (5-6), pp. 627-630.

Ruch, W., Altfreder, O., \& Proyer, R. T. (2009). 'How do gelotophobes interpret laughter in ambiguous situations? An experimental validation of the concept'. Humor: International Journal of Humor Research 22 (1-2), pp. 63-89.

Ruch, W. F., Hofmann, J., \& Platt, T. (2013). 'Investigating facial features of four types of laughter in historic illustrations'. The European Journal of Humour Research 1 (1), pp. 99-118.

Ruch, W., Hofmann, J., Platt, T., \& Proyer, R. T. (2014). 'The state-of-the art in gelotophobia research: A review and some theoretical extensions'. Humor: International Journal of Humor Research 27 (1), pp. 23-45.

Ruch, W., Hofmann, J., \& Platt, T. (2015). 'Individual differences in gelotophobia and responses to laughter-eliciting emotions'. Personality and Individual Differences 72, pp. 117-121.

Samson, A. C., Huber, O., \& Ruch, W. (2011). 'Teasing, ridiculing and the relation to the fear of being laughed at in individuals with Asperger's syndrome'. Journal of Autism and Developmental Disorders 41 (4), pp. 475-483.

Sternberger, R. T., Turner, S. M., Beidel, D. C., \& Calhoun, K. S. (1995). 'Social phobia: an analysis of possible developmental factors'. Journal of Abnormal Psychology 104 (3), pp. 526-531. 
Sue, S., McKinney, H. L., \& Allen, D. B. (1976). 'Predictors of the duration of therapy for clients in the community mental health system'. Community Mental Health Journal 12 (4), pp. 365-375.

Temerlin, M. K. (1956). 'One determinant of the capacity to free-associate in psychotherapy'. The Journal of Abnormal and Social Psychology 53 (1), pp. 16-18.

Titze, M. (1996). 'The Pinocchio complex: Overcoming the fear of laughter'. Humor \& Health Journal 5, pp. 1-11.

Titze, M. (2009). 'Gelotophobia: The fear of being laughed at'. Humor: International Journal of Humor Research 22 (1-2), pp. 27-48.

Ventis. W.L. \& Platt, T. (2014). 'Why have I never treated a gelotophobic?' Paper presented at the International Society for Humor Studies Conference, Utrect, Netherlands, July 7-11, 2014.

Weiss, E. M., Schulter, G., Freudenthaler, H. H., Hofer, E., Pichler, N., \& Papousek, I. (2012). 'Potential markers of aggressive behaviour: the fear of other persons' laughter and its overlaps with mental disorders'. PloS one 7 (5), e38088. 